\title{
Pacific
}

Journal of

Mathematics

\section{A NOTE ON THE SET OF PERIODS FOR KLEIN BOTTLE MAPS}

JAUME LLIBRE 


\title{
A NOTE ON THE SET OF PERIODS FOR KLEIN BOTTLE MAPS
}

\section{JAUME LLIBRE}

\begin{abstract}
By using the Nielsen fixed point theory we characterize the set of periods attained by continuous self-maps on the Klein bottle belonging to a given homotopy class.
\end{abstract}

1. Introduction. In dynamical systems, it is often the case that topological information can be used to study qualitative and quantitative properties (like the set of periods) of the system. This note deals with the problem of determining the set of periods (of the periodic points) of a continuous self-map of the Klein bottle. Our interest in this problem comes from the fact that the unique manifolds in dimensions 1 and 2 with zero Euler characteristic are the circle, torus and Klein bottle, and for the first two the structure of the set of periods of their continuous self-maps has been determined.

To fix terminology, suppose $f$ is a continuous self-map on the manifold $M$. A fixed point of $f$ is a point $x$ in $M$ such that $f(x)=x$. We shall call $x$ a periodic point of period $n$ if $x$ is a fixed point of $f^{n}$ but is not fixed by any $f^{k}$, for $1 \leq k<n$. We denote by $\operatorname{Per}(f)$ the set of natural numbers corresponding to periods of periodic orbits of $f$.

Even for continuous self-maps $f$ on the circle the relation between the degree of $f$ and the set $\operatorname{Per}(f)$ is interesting and nontrivial (see [5], [3] and for more details [2]). Let $\mathbb{N}$ denote the set of natural numbers. Suppose $f$ has degree $d$.

(1) For $d \notin\{-2,-1,0,1\}, \operatorname{Per}(f)=\mathbb{N}$.

(2) For $d=-2, \operatorname{Per}(f)$ is either $\mathbb{N}$ or $\mathbb{N} \backslash\{2\}$.

(3) For $d=-1,0, \operatorname{Per}(f) \supset\{1\}$.

(4) For $d=1$, the set $\operatorname{Per}(f)$ can be empty.

Recently, in [1] these results have been extended to continuous self-maps on the 2-dimensional torus, and many of them to the $m$ dimensional torus with $m>2$.

The goal of this note is to provide a similar description of the set of periods for continuous self-maps on the Klein bottle, or simply Klein bottle maps. 
Let $K$ be the Klein bottle and let $f$ be a Klein bottle map. If $p$ is a base point for $K$, then $f$ is homotopic to a continuous map $h$, $f \sim h$, such that $h(p)=p$ and a presentation of the group $\Pi(K, p)$ is given by two generators $a$ and $b$ and the relation $a b a b^{-1}=1$. Halpern in [6] shows that $h_{p}(a)=a^{u}$ and $h_{p}(b)=a^{w} b^{v}$ for some integers $u, v$ and $w$, where $h_{p}$ is the induced endomorphism on $\Pi(K, p)$. In what follows $u$ and $v$ will be called the integers associated to $f$.

Now our main result can be stated as follows. Here, $2 \mathbb{N}$ denotes the set of all even natural numbers.

THEOREM. Let $f$ be a Klein bottle map and let $u$ and $v$ its associated integers.

(1) Suppose $|u|>1$. Then

$$
\begin{array}{ll}
\operatorname{Per}(f)=\mathbb{N} & \text { if }|v| \neq 1, \text { and } \\
\operatorname{Per}(f) \supset \mathbb{N} \backslash 2 \mathbb{N} & \text { if } v=-1 .
\end{array}
$$

Furthermore, the last result cannot be improved without additional hypothesis on $f$.

(2) Suppose $|u| \leq 1$. Then

$$
\begin{array}{ll}
\operatorname{Per}(f)=\mathbb{N} & \text { if } v \notin\{-2,-1,0,1\}, \\
\operatorname{Per}(f) \supset \mathbb{N} \backslash\{2\} & \text { if } v=-2, \\
\operatorname{Per}(f) \supset\{1\} & \text { if } v \in\{-1,0\} .
\end{array}
$$

Furthermore, these last two results cannot be improved without additional hypotheses on $f$.

(3) If $v=1$ then the set $\operatorname{Per}(f)$ can be empty.

This theorem will be proved in the next section. Its proof is based in the results of Halpern [6] on the Nielsen numbers of Klein bottle maps. Unfortunately this nice paper of Halpern remains unpublished.

As we shall see the Nielsen numbers of the Klein bottle map $f$ only depend on its associated integers. Then, since the Nielsen numbers are homotopy invariants, the characterization of the set of periods given in the theorem works for all Klein bottle maps homotopic to $f$.

I thank Christopher McCord for his comments on the Nielsen fixed point theory. 
2. Periods and Nielsen numbers. We start by recalling the definition of Nielsen number, for more details see, for instance, [4], [7] or [8]. Suppose that $f: E \rightarrow E$ is a continuous map of a compact ENR. The Nielsen number $N(f)$ is defined as follows. First an equivalance relation $\sim$ is defined on the set $F$ of fixed points of $f$. Two fixed points $x, y$ are equivalent, $x \sim y$, provided there is a path $\gamma$ in $E$ from $x$ to $y$ such that $f \circ \gamma$ and $\gamma$ are homotopic and the homotopy fixes endpoints. The set of equivalence classes $F / \sim$ is known to be finite and each equivalence class is compact. Each of these equivalence classes will be called a fixed point class. Using a fixed point index, we may assign an index to each fixed point class. The Nielsen number is defined as the number of fixed point classes with nonzero index.

Notice that from the definition of Nielsen number, $f$ has at least $N(f)$ fixed points. If $g \sim f$ then $g$ has at least $N(f)$ fixed points, and $N(g)=N(f)$.

In what follows $f$ will be a Klein bottle map, and $u$ and $v$ its associated integers. Halpern in [6] proves that

$$
N\left(f^{n}\right)= \begin{cases}\left|u^{n}\left(v^{n}-1\right)\right| & \text { if }|u|>1 \\ \left|v^{n}-1\right| & \text { if }|u| \leq 1\end{cases}
$$

for all $n \geq 1$.

Define $A_{n}$ for $n \geq 1$ inductively as follows. For $u \neq 1$, set

$$
A_{1}=N(f)
$$

and

$$
A_{n}=N\left(f^{n}\right)-\sum_{k<n, k \mid n} A_{k} \quad \text { for } n>1,
$$

where $k \mid n$ denotes $k$ divides $n$.

For $u=1$, set

$$
A_{1}=N(f)
$$

and

$$
A_{n}=N\left(f^{n}\right)-\sum_{k<n, k \mid n, \frac{n}{k} \text { odd }} A_{k} \quad \text { for } n>1 .
$$

For a Klein bottle map $g$ and $n \geq 1$, let $P_{n}(g)$ be the set of periodic points of $g$ of period $n$. The cardinality of a set $S$ is denoted by $\operatorname{Card}(S)$. Halpern also shows that

$$
\min \left\{\operatorname{Card}\left(P_{n}(g)\right): g \sim f\right\}=A_{n} \quad \text { for all } n \geq 1 .
$$

In particular, from (b) it follows that $A_{n} \geq 0$ for all $n \geq 1$. 
Furthermore, except for two special cases $u \geq 1$ and $v=-1$, or $u=1$ and $|v|>1$, Halpern shows that there exists a Klein bottle map $g \sim f$ such that

$$
\operatorname{Card}\left(P_{n}(g)\right)=A_{n} \quad \text { for all } n \geq 1 .
$$

Finally, he shows for each $m \geq 1$ that there exists a Klein bottle map $g \sim f$ such that

$$
\operatorname{Card}\left(P_{n}(g)\right)=A_{n} \quad \text { for } 1 \leq n \leq m .
$$

The next proposition plays a main role in the proof of Theorem.

Proposition. The following statements hold.

(1) Suppose $|u|>1$. If $|v| \neq 1$ then

$$
\sum_{k<n, k \mid n} N\left(f^{k}\right)<N\left(f^{n}\right)
$$

for all $n>1$. If $v=-1$ then (e) holds for all odd $n>1$.

(2) Suppose $|u| \leq 1$. If $v \notin\{-2,-1,0,1\}$ then (e) holds for all $n>1$. If $v=-2$ then (e) holds for all $n>2$.

Proof. Suppose $|u|>1$. We separate the proof of (1) into four cases.

Case 1. $v=0$. From (a) we obtain (e) as follows:

$$
\sum_{k=1}^{n-1} N\left(f^{k}\right)=\sum_{k=1}^{n-1}|u|^{k}=\frac{|u|^{n}-|u|}{|u|-1}<|u|^{n}=N\left(f^{n}\right) .
$$

Case 2. $v=-1$. Then $N\left(f^{n}\right)$ is 0 for $n$ even, and $2|u|^{n}$ for $n$ odd. Hence, for $n$ odd the proof of (e) follows as in Case 1.

Case 3. $v>1$. For $n>1$ we have

$$
\begin{aligned}
\sum_{k=1}^{n-1} N\left(f^{k}\right) & =\sum_{k=1}^{n-1}\left(|u|^{k} v^{k}-|u|^{k}\right)=\frac{|u|^{n} v^{n}-|u| v}{|u| v-1}-\frac{|u|^{n}-|u|}{|u|-1} \\
& <\frac{|u|^{n} v^{n}-|u| v-|u|^{n}+|u|}{|u| v-1}<\frac{|u|^{n} v^{n}-|u|^{n}}{|u| v-1} \\
& <|u|^{n} v^{n}-|u|^{n}=N\left(f^{n}\right) .
\end{aligned}
$$


Case 4. $v<-1$. For $n>1$ we have

$$
\begin{aligned}
\sum_{k=1}^{n-1} N\left(f^{k}\right) & =\sum_{k=1}^{n-1}|u|^{k}\left|v^{k}-1\right|=\sum_{k=1}^{n-1}|u v|^{k}+\sum_{k=1}^{n-1}(-1)^{k+1}|u|^{k} \\
& =\frac{|u v|^{n}-|u v|}{|u v|-1}+\frac{|u|+(-1)^{n}|u|^{n}}{1+|u|}
\end{aligned}
$$

Therefore, if $n>1$ is odd, clearly we have

$$
\sum_{k=1}^{n-1} N\left(f^{k}\right)<|u v|^{n}+|u|^{n}=N\left(f^{n}\right)
$$

and if $n>2$ is even, we have

$$
\begin{aligned}
\sum_{k=1}^{n-2} N\left(f^{k}\right) & =\frac{|u v|^{n-1}-|u v|}{|u v|-1}+\frac{|u|+(-1)^{n-1}|u|^{n-1}}{1+|u|} \\
& <|u v|^{n-1}+|u|^{n-1}=|u|^{n-1}\left(|v|^{n-1}+1\right) \\
& <|u|^{n}\left(|v|^{n}-1\right)=N\left(f^{n}\right) .
\end{aligned}
$$

So (e) holds for all $n>2$. Suppose $n=2$. Since

$$
N(f)=|u|(|v|+1)<|u|^{2}\left(|v|^{2}-1\right)=N\left(f^{2}\right),
$$

(e) also holds for $n=2$. This completes the proof of (1).

Suppose $|u| \leq 1$. Then, from $(\mathrm{a}), N\left(f^{n}\right)=\left|v^{n}-1\right|$. We separate the proof of (2) into two cases.

Case 1: $v \geq 2$. Then for $n>1$ we have

$$
\sum_{k=1}^{n-1}\left|v^{k}-1\right|=\frac{v^{n}-v}{v-1}-(n-1)<v^{n}-1<\left|v^{n}-1\right|,
$$

and (e) follows.

Case 2: $v \leq-2$. We have

$$
\left|v^{k}-1\right|= \begin{cases}|v|^{k}-1 & \text { for } k \text { even } \\ |v|^{k}+1 & \text { for } k \text { odd }\end{cases}
$$

Therefore for $n$ odd we have

$$
\sum_{k=1}^{n-1}\left|v^{k}-1\right|=\frac{|v|^{n}-|v|}{|v|-1}<|v|^{n}+1=\left|v^{n}-1\right|,
$$


and (e) follows. If $n$ is even then for $v \leq-3$ we have

$$
\sum_{k=1}^{n-1}\left|v^{k}-1\right|=\frac{|v|^{n}-|v|}{|v|-1}+1<|v|^{n}-1=\left|v^{n}-1\right|,
$$

and (e) follows.

If $n$ is even and $v=-2$ we get

$$
\sum_{k=1}^{n-1}\left|v^{k}-1\right|=\frac{2^{n}-2}{2-1}=2^{n}-1=\left|v^{n}-1\right| .
$$

However, if $n>2$ then there exists $k \in\{1,2, \ldots, n-1\}$ which does not divide $n$. For this $k$ we have $\left|v^{k}-1\right|>0$, so (e) also holds.

The proof of statement (2) of Proposition 1 is essentially the proof for continuous self-maps on the circle, because for such maps the Nielsen number is $\left|d^{n}-1\right|$ where $d$ denotes the degree of the maps, see for instance [2].

Proof of the Theorem. From the definition of the numbers $A_{k}$, clearly we have $A_{k} \leq N\left(f^{k}\right)$ for all $k \geq 1$. Therefore, if (e) is true for some $n>1$, we have, for this $n$, that

$$
A_{n}=N\left(f^{n}\right)-\sum_{k<n, k \mid n} A_{k} \geq N\left(f^{n}\right)-\sum_{k<n, k \mid n} N\left(f^{k}\right)>0
$$

when $u \neq 1$, and

$$
\begin{aligned}
A_{n} & =N\left(f^{n}\right)-\sum_{k<n, k \mid n, \frac{n}{k} \text { odd }} A_{k} \geq N\left(f^{n}\right)-\sum_{k<n, k \mid n, \frac{n}{k} \text { odd }} N\left(f^{k}\right) \\
& \geq N\left(f^{n}\right)-\sum_{k<n, k \mid n} N\left(f^{k}\right)>0
\end{aligned}
$$

when $u=1$. From (b) it follows that $\operatorname{Per}(f) \supset\left\{n \in N: A_{n}>0\right\}$. Hence, by the proposition we obtain the first part of statements (1) and (2) of the theorem.

From (c) and (d) the second part of statements (1) and (2), and statement (3) follow immediately.

\section{REFERENCES}

[1] L. Alsedà, S. Baldwin, J. Llibre, R. Swanson and W. Szlenk, Minimal sets of periods for torus maps via Nielsen numbers, submitted, 1991.

[2] L. Alsedà, J. Llibre and M. Misiurewicz, Combinatorial dynamics and entropy in dimension one, submitted, 1990. 
[3] L. Block, J. Guckenheimer, M. Misiurewicz and L. S. Young, Periodic Points and Topological Entropy of One Dimensional Maps, Springer Lecture Notes in Math., 819 (1980), 18-34.

[4] R. Brown, The Lefschetz Fixed Point Theorem, Scott, Foresman and Company, 1971.

[5] L. S. Efremova, Periodic orbits and a degree of a continuous map of a circle (in Russian), Diff. and Integer Equations (Gor'kii), 2 (1978), 109-115.

[6] B. Halpern, Periodic points on the Klein bottle, preprint, 1979.

[7] B. Jiang, Lectures on Nielsen fixed point theory, Contemporary Math., 14 (1983).

[8] T. H. Kiang, The Theory of Fixed Point Classes, Springer, 1989.

Received May 13, 1991. Partially supported by a DGICYT grant number PB90-0695.

NORTHWESTERN UNIVERSITY

EVANSTON, IL 60208-2730

Universitat Autònoma de Barcelona

08193 BELLATERRA

BARCELONA, SPAIN 



\title{
PACIFIC JOURNAL OF MATHEMATICS
}

Founded by

\author{
E. F. Beckenbach (1906-1982) F. Wolf (1904-1989)
}

\section{EDITORS}

V. S. VARADARAJAN

(Managing Editor)

University of California

Los Angeles, CA 90024-1555

vsv@math.ucla.edu

Herbert Clemens

University of Utah

Salt Lake City, UT 84112

clemens@math.utah.edu

F. Michael Christ

University of California

Los Angeles, CA 90024-1555

christ@math.ucla.edu

THOMAS ENRIGHT

University of California, San Diego

La Jolla, CA 92093

tenright@ucsd.edu
Nicholas ERcolani

University of Arizona

Tucson, AZ 85721

ercolani@math.arizona.edu

R. FInN

Stanford University

Stanford, CA 94305

finn@gauss.stanford.edu

VAughan F. R. Jones

University of California

Berkeley, CA 94720

vfr@math.berkeley.edu

STEVEN KERCKHOFF

Stanford University

Stanford, CA 94305

spk@gauss.stanford.edu

\section{C. MOORE}

University of California

Berkeley, CA 94720

MARTIN SCHARLEMANN

University of California

Santa Barbara, CA 93106

mgscharl@henri.ucsb.edu

Harold Stark

University of California, San Diego La Jolla, CA 92093

\section{SUPPORTING INSTITUTIONS}

UNIVERSITY OF ARIZONA

UNIVERSITY OF BRITISH COLUMBIA

CALIFORNIA INSTITUTE OF TECHNOLOGY

UNIVERSITY OF CALIFORNIA

MONTANA STATE UNIVERSITY

UNIVERSITY OF NEVADA, RENO

NEW MEXICO STATE UNIVERSITY

OREGON STATE UNIVERSITY
UNIVERSITY OF OREGON

UNIVERSITY OF SOUTHERN CALIFORNIA

STANFORD UNIVERSITY

UNIVERSITY OF HAWAII

UNIVERSITY OF TOKYO

UNIVERSITY OF UTAH

WASHINGTON STATE UNIVERSITY

UNIVERSITY OF WASHINGTON 


\section{PACIFIC JOURNAL OF MATHEMATICS}

Volume $157 \quad$ No. $1 \quad$ January 1993

Permutation enumeration symmetric functions, and unimodality

FRANCESCO BRENTI

On the analytic reflection of a minimal surface

JAIGYOUNG CHOE

Contractive zero-divisors in Bergman spaces

Peter Larkin DUREn, DMitry Khavinson, Harold SEymour

SHAPIRO and CARL SUNDBERG

On the ideal structure of positive, eventually compact linear operators on

Banach lattices

RUEY-JEN JANG and HAROLD DEAN VICTORY, JR.

A note on the set of periods for Klein bottle maps

JAUME LLIBRE

Asymptotic expansion at a corner for the capillary problem: the singular case

\section{ERICH MIERSEMANN}

A state model for the multivariable Alexander polynomial

JUN MURAKAMI

Free Banach-Lie algebras, couniversal Banach-Lie groups, and more

Vladimir G. Pestov

Four manifold topology and groups of polynomial growth

RICHARD ANDREW STONG

A remark on Leray's inequality

AKIRA TAKESHITA

$A_{\infty}$ and the Green function

JANG-MEI GLORIA WU

Integral spinor norms in dyadic local fields. I 